\title{
Proceeding
}

Supplementary Issue: Autumn Conferences of Sports Science. Costa Blanca Sports Science Events, 18-19 December 2020. Alicante, Spain.

\section{Effect of internal focus of attention with touching cue on the agonist muscle activity during exercise}

\author{
KAZUSHIGE OSHITA \\ Department of Human Information Engineering, Okayama Prefectural University, Soja-shi, Japan
}

\begin{abstract}
This study aimed to investigate the effect of internal focus of attention with touching cue on agonist muscular activity during the arm curl exercise. Ten healthy males (age, 20-35 years) performed five repetitions of arm curl under three different focus instructions: external focus condition (EXT), where participants were instructed to concentrate on lifting the bar; internal focus condition (INT), where participants were instructed to concentrate on contractions of biceps brachii; and, internal focus and touch condition (INT+T): participants' biceps brachii was lightly touched by the investigator and they were instructed to concentrate on muscle contractions at the touched point. Although the average muscular activities in INT and INT+T were significantly higher than that of EXT $(p<.01)$, no significant difference in muscle activity between INT and INT+T was observed. Further, magnitudes of these effects were small $(d<0.25)$. These results suggest that internal focus caused by individual's consciousness, as well as a focus attention by touching the agonist area increased the agonist muscle activity during exercise. However, the increase in agonist muscle activity due to focus attention is limited.
\end{abstract}

Keywords: External focus attention; Light touch effect; Resistance training; Exercise instructor.

\section{Cite this article as:}

Oshita, K. (2021). Effect of internal focus of attention with touching cue on the agonist muscle activity during exercise. Journal of Human Sport and Exercise, 16(2proc), S195-S201. doi:https://doi.org/10.14198/jhse.2021.16.Proc2.04

Corresponding author. Department of Human Information Engineering, Okayama Prefectural University, Soja-shi, Japan. https://orcid.org/0000-0002-8049-1174

E-mail: oshita@ss.oka-pu.ac.jp

Abstract submitted to: Autumn Conferences of Sports Science. Costa Blanca Sports Science Events, 18-19 December 2020. Alicante, Spain.

JOURNAL OF HUMAN SPORT \& EXERCISE ISSN 1988-5202

(c) Faculty of Education. University of Alicante

doi:10.14198/jhse.2021.16.Proc2.04 


\section{INTRODUCTION}

Muscle activity during exercise differs depending on an individual's focus attention when lifting an external resistance, even with the same load of resistance (Neumann, 2019; Schoenfeld and Contreras, 2016). In the case of resistance training, internal focus of attention (INT) means the individual concentrates on contracting the agonist muscle when lifting a weight, while external focus of attention (EXT), on lifting the weight. Previous reviews (Neumann, 2019; Schoenfeld and Contreras, 2016) reported that although higher electromyographic (EMG) activity in the agonist muscle is observed in INT as compared to EXT, movement velocity (including angular velocity or power production) during exercise is executed faster in EXT than in INT. Therefore, these previous studies suggest that if muscle strength gain or muscle hypertrophy gain is the desired goal, then INT should be used during exercise. However, if individuals desire to improve movement performance, and speed, it is argued that they should adopt EXT.

Increased muscular activity observed in INT indicates that understanding of agonist muscle group during exercise is an important factor in enhancing the effect of resistance training. However, individuals (especially in novice of resistance training) face difficulties in understanding the agonist muscle during resistance training (Oshita et al., 2016a; 2016b; 2019), even in simple single-joint exercise (Oshita et al., 2018), or well-known stretching exercise (Oshita et al., 2017). To enhance understanding of the agonist muscle, the instructor occasionally touches the agonist muscle area, and instructs the client with cues such as "concentrate on contracting of this (touching) part" during exercise. Some researchers have reported effectiveness of touching cue; lightly touching the body part can enhance the perception of body orientation, and this influence human movement (Boonsinsukh et al., 2009; Oshita and Yano, 2015; 2016; 2017; 2020; Stramel et al., 2019). Previous study reported that such an internal focus caused by a touching stimuli (INT+T) to the agonist area increased the agonist muscle activity during exercise as compared to EXT (Oshita, in press). This result proves that touching cue from an instructor is effective in increasing agonist muscle activity during exercise.

While INT is focus of the individual's subjective consciousness only, INT+T adds the focus of being touched at agonist region in addition to the subjective focus. It seems that the individual can now concentrate more on muscle contraction. If the individual achieves more concentration on muscle contraction during INT+T than INT, agonist muscle activity will increase in INT+T as compared to INT. Therefore, this study investigated the effect of INT with touching cue on agonist muscular activity during the arm curl exercise.

\section{MATERIALS AND METHODS}

\section{Participants}

Data were collected from 10 healthy males (age, 20-35 years) with no current or previous medical history of musculoskeletal system disorders. Each participant identified the preferred dominant arm they used for writing. This was the right side for all the participants. All participants provided informed consent after the details of this experiment were explained. This study was approved by the Human Ethics Committee of the Graduate School of Human Development and Environment, Kobe University.

\section{Procedures}

Arm curl exercises were conducted using a preacher-curl machine on the non-preferred arm of the participants. Muscular activity in the biceps brachii was evaluated during the exercise. Surface EMG data were collected from the biceps brachii muscles to assess muscular activity. On the non-preferred arm, bipolar surface electrodes with an EMG amplifier (ID2PAD; Oisaka Electronic Equipment Ltd., Japan) were placed over the muscle belly at 2-cm inter-electrode distance. To decrease skin impedance affecting the electrical 
signal, the skin was wiped by gentle abrasion with a skin preparation gel and isopropyl alcohol swabs. The signals were amplified 500 times and acquired at sampling frequency of $1 \mathrm{kHz}$ using a data logger with an analogue-to-digital converter (LP-MS1002; Logical Product Corporation, Japan).

\section{Experimental protocol}

Maximum voluntary muscle isometric contraction (MVC) test was initially performed in the direction of elbow flexion at $90^{\circ}$ joint angle. The participant sat on a seat of the preacher-curl machine while his non-preferred arm held a bar. The bar was fixed to the ground by a metal chain through a strain gauge (FG-5100, Lutron Electronic Enterprise Co., Ltd., Taiwan). Participants were instructed to attempt to flex their arm against the fixed bar. During the MVC tests, the visual signals depicting force were displayed on a digital indicator and verbal encouragement was provided by investigators. Participants were asked to perform the MVC test twice for $5 \mathrm{~s}$ with a $30 \mathrm{~s}$ pause in between the tests. The participants were allowed to reject an effort they deemed "sub-maximal." The highest peak force was selected as the MVC force.

After the MVC tests, the participants performed an arm curl task weighted with a dumbbell. Mass of the dumbbell was equivalent to $50 \%$ of the MVC force. Participants performed five repetitions of arm curl with full range of motion under three different focus instructions, with $5 \mathrm{~min}$ of resting period between the sets. For EXT, participants were instructed to concentrate on lifting the dumbbell, whereas for the INT, participants were instructed to concentrate on the contractions of biceps brachii. For the INT+T, the participants' biceps brachii was lightly touched by the investigator and they were instructed to concentrate on muscle contractions at the touched point (Figure 1). During these tasks, participants were asked to look straight ahead and concentrate on the bar, agonist muscle contractions or contractions of the touched point. The movement time of the arm curl was prescribed by using a digital metronome (MA-1, KORG Inc., Japan) set to produce a click every $1.5 \mathrm{~s}$. Participants were asked to perform their movements in synchrony with the metronome signal, i.e., the end of each upward and downward motion should coincide with one signal. The order of focus conditions was randomized across all the participants. The weight mass and movement tempo of this study were based on a previous study that investigated the effects of focus of attention on muscle activity during arm curl (Oshita et al., in press; Vance et al., 2004). Before the MVC tests, a practice session to familiarize the participants with the arm curl task was conducted for approximately 5 min without dumbbell.
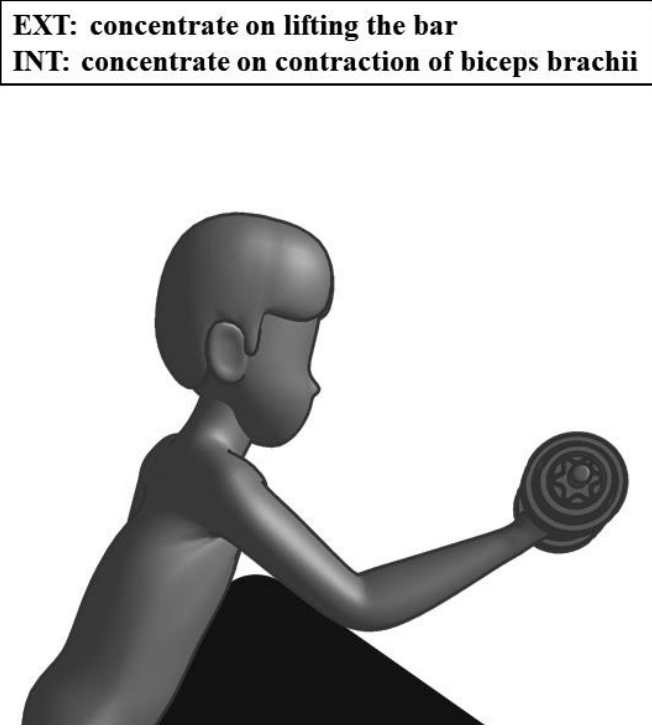

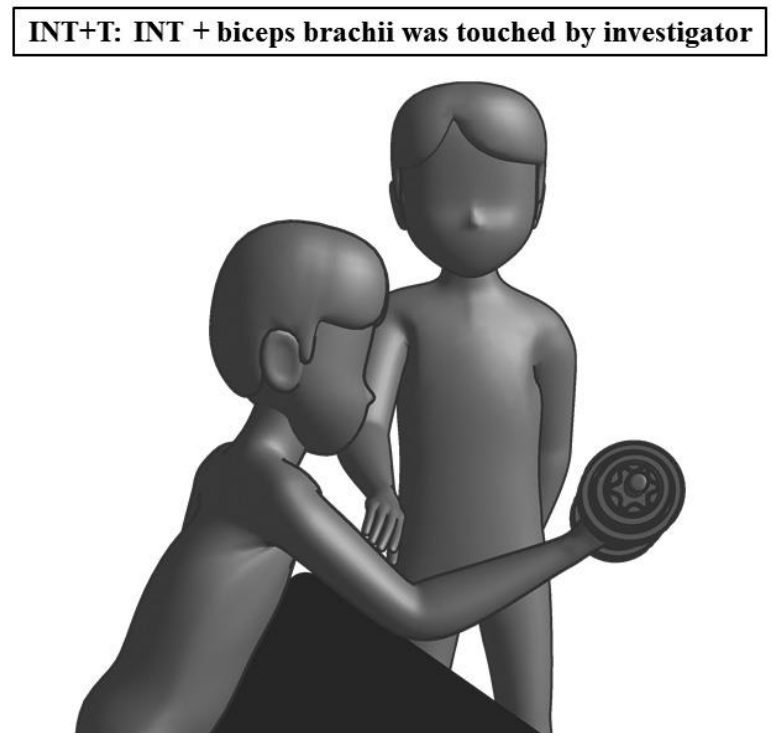

Figure 1. Schematic diagrams of the arm-curl experiment in three focus attention conditions. 


\section{Data and statistical analysis}

The stored EMG data were processed using the waveform analysis software SPCANA (ver. 4.92). After bandpass filtering (1-500 Hz), the root mean square of the EMG signal (RMS-EMG) in each task was calculated. For the MVC task, RMS-EMG was calculated at every $0.2 \mathrm{~s}$, and the maximum $0.2 \mathrm{~s}$ interval RMS-EMG value from the two MVCs was taken as the MVC value. In each arm curl task, a period of 0.5 seconds in the middle portion in each upward portion was selected to calculate the RMS-EMG value. 2nd-4th repetitions in each focus condition were selected for analysis, and the average RMS-EMG value was calculated. The average RMS-EMGs in each arm curl task were normalized to the RMS-EMG in the MVC task (\%MVC) for muscle activity evaluation.

Muscle activities (\%MVC) in each arm curl task were evaluated using one-way repeated-measures analysis of variance (ANOVA), and differences between the three different focus were evaluated with post-hoc multiple comparisons using Tukey's test. Furthermore, the magnitude of the effect (Cohen's $d$ ) of \%MVC between the two focus conditions was calculated.

Value of $p<.05$ was considered statistically significant. These analyses were performed using JSTAT (ver. $20.0 \mathrm{~J})$ software. Data were presented as mean values \pm standard errors of mean (SEM), unless otherwise stated.

\section{RESULTS}

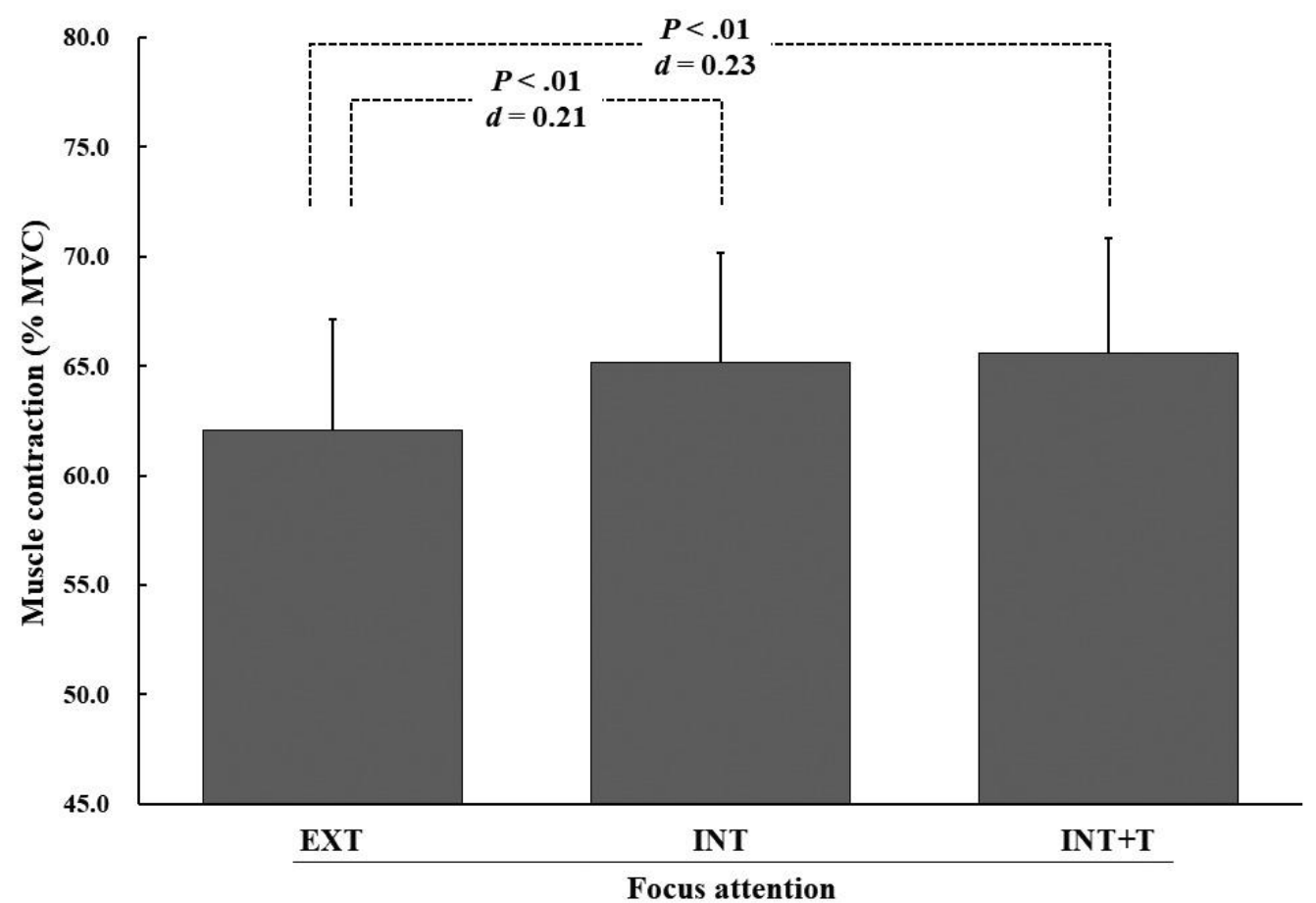

Figure 2. Muscle activities in the biceps brachii during arm-curl exercise under three focus attention conditions

The average \%MVC during arm curl tasks in the EXT, INT, and INT+T focus conditions were $62.04 \pm 5.09 \%$, $65.18 \pm 4.95 \%$, and $65.58 \pm 5.23 \%$, respectively (Figure 2). The average \%MVC in the INT and INT+T focus conditions were significantly higher than that of the EXT focus condition $(p<.01)$. However, the effect size compared to EXT was small for both INT $(d=0.21)$ and INT+T $(d=0.23)$. No significant difference of \%MVC 
between INT and INT+T focus condition was observed, and magnitude of effect was small $(d=0.03)$. These results indicate that although internal focus caused by an individual's consciousness, as well as focus of attention by touching the agonist area slightly increased the agonist muscle activity, there was no significant difference in muscle activity among different methods of internal focus (i.e., an individual's consciousness or a touch cueing).

\section{DISCUSSION}

The average \%MVC in the INT and INT+T focus conditions were significantly higher than that in the EXT condition. Increased agonist muscular activity is observed in the INT as compared to the EXT is consistent with the results of previous studies (Neumann, 2019; Oshita et al., in press; Schoenfeld and Contreras, 2016; Vance et al., 2004). Although antagonist muscle activity was not measured in this study, a previous study reported that associated increase in the antagonist muscle activity was in line with the agonist muscle activity during INT. This suggests an association between the antagonist and agonist muscles during human movements (Vance et al., 2004). As a result, force output becomes difficult by this association when INT is performed, and the agonist muscle activity might increase. An increased voluntary muscular activity not accompanied with gross force or movement output suggests an increase in "noise" in the motor control network (Marchant et al., 2005; Vance et al., 2004; Zachry et al., 2005). Furthermore, this study revealed that INT caused by an individual's consciousness, as well as focus of attention by touching the agonist area, increased the agonist muscle activity.

While INT is only an individual's own consciousness, INT+T adds a touching stimulus to the consciousness. Although it seems that INT+T is easier for concentration on the agonist area as compared to INT, no significant difference in muscle activity was observed between INT and INT+T focus conditions and the magnitude of their effect was slight. Therefore, it may be concluded that an increase in agonist muscle activity due to focus conditions is limited. On the other hand, one participant in the present experiment told the investigator that it was hard for him to concentrate on muscle contractions because he was distracted by the touching. This comment makes us speculate that some individuals may not be able to utilize touch stimulation effectively. In the actual exercise instruction, some instructor touches clients' agonist muscle area only in relaxation phase of the exercise (does not touch in contraction phase). Although the participants' biceps brachii was touched by the investigator all the time during arm curl exercise in the present study, this instruction method suggests that touching timing might influence muscle activity. Because the effect of muscle activity might differ depending such a touching method, further research is essential to examine the details of touch stimuli, including timing, pressure, size of area, and so on.

Hence, the present study demonstrates that internal focus caused by individual's consciousness, as well as a focus attention by touching the agonist area is also effective in increasing muscle activity during exercise. Although individuals lacking resistance training face difficulties in understanding the agonist muscle during resistance exercise (Oshita et al., 2016a; 2016b; 2018; 2019), the agonist muscle area could be touched to effectively enhance the agonist muscle activity and direct the focus of attention to the agonist muscle.

\section{CONCLUSION}

This study investigated the effect of INT with touching cue on agonist muscular activity during the arm curl exercise. Arm curl exercises under three different focus instructions were performed: external focus condition (EXT), where participants were instructed to concentrate on lifting the bar; internal focus condition (INT), where participants were instructed to concentrate on contractions of biceps brachii; and, internal focus and 
touch condition (INT+T): participants' biceps brachii was lightly touched by the investigator and they were instructed to concentrate on muscle contractions at the touched point. Although the average \%MVC in the INT and INT+T focus conditions were significantly higher than that in the EXT condition $(p<.01)$, no significant difference in muscle activity between INT and INT+T was observed. Further, magnitudes of these effects were small $(d<0.25)$. These results suggest that internal focus caused by individual's consciousness, as well as a focus attention by touching the agonist area increased the agonist muscle activity during exercise. However, the increase in agonist muscle activity due to focus attention is limited.

\section{SUPPORTING AGENCIES}

Research supported by KAKENHI (Grant-in-Aid for Scientific Research C, Grant Number JP18K06455).

\section{DISCLOSURE STATEMENT}

The author declares no conflict of interest.

\section{REFERENCES}

Boonsinsukh, R., Panichareon, L., \& Phansuwan-Pujito, P. (2009). Light touch cue through a cane improves pelvic stability during walking in stroke. Archives of physical medicine and rehabilitation, 90(6), 919-926. https://doi.org/10.1016/j.apmr.2008.12.022

Marchant, D. C., Greig, M., Bullough, J., \& Hitchen, D. (2011). Instructions to adopt an external focus enhance muscular endurance. Research quarterly for exercise and sport, 82(3), 466-473. https://doi.org/10.1080/02701367.2011.10599779

Neumann, D.L. (2019). A Systematic review of attentional focus strategies in weightlifting. Frontiers in Sports and Active Living, 1, 7. https://doi.org/10.3389/fspor.2019.00007

Oshita, K. \& Yano, S. (2015). The Effect of Lightly Gripping a Cane on the Dynamic Balance Control. The open biomedical engineering journal, 9, 146-50. https://doi.org/10.2174/1874120701509010146

Oshita, K., \& Yano, S. (2016). Effect and immediate after-effect of lightly gripping the cane on postural sway. Journal of physiological anthropology, 35(1), 14. https://doi.org/10.1186/s40101-016-0096-4

Oshita, K., Hagiwara, G., Monma, T., Tsuno, T., Koizumi, K., Oyama, Y., Yamaguchi, K., Tashiro, T. \& Funatsu, K. (2016a). Association Between Previous Experience of Strength Training Under Appropriate Supervision and Knowledge of Squats Among University Students who Exercise Regularly. International Journal of Sport and Health Science, 14, 61-71. https://doi.org/10.5432/ijshs.201606

Oshita, K., Hagiwara, G., Monma, T., Tsuno, T., Koizumi, K., Oyama, Y., Yamaguchi, K., Tashiro, T., Funatsu, K. \& Ariyoshi, K. (2016b). Consciousness of training body part(s) by showing a picture of an exercise to young inexperienced people: A case of squatting exercises. Japanese Journal of Physical Fitness and Sports Medicine, 65(4), 421-429. https://doi.org/10.7600/ispfsm.65.421

Oshita, K., \& Yano, S. (2017). Effect of haptic sensory input through a fluttering cloth on tandem gait performance. Human movement science, 55, 94-99. https://doi.org/10.1016/j.humov.2017.08.008

Oshita, K., Matsuzaki, T., Hikita, A., Miyazaki, R., Hagiwara, G., Tashiro, T., Tsuno, T., Higuchi, Y. \& Funatsu, K. (2017). Association between Experience of Supervised Strength Training and the Knowledge of Stretching. Gazzetta Medica Italiana Archivio per le Scienze Mediche, 176 (11), 613624. https://doi.org/10.23736/S0393-3660.16.03422-7 
Oshita, K., Matsuzaki, T., Hikita, A., Miyazaki, R., Hagiwara, G., Tashiro, T. \& Higuchi, Y. (2018). A study of body-part(s) training consciousness using a picture of a single-joint exercise. Japanese Journal of Physical Fitness and Sports Medicine, 67(6), 393-401. https://doi.org/10.7600/jspfsm.67.393

Oshita, K., Matsuzaki, T., Hikita, A., Miyazaki, R., Hagiwara, G., Tashiro, T., Tsuno, T., Higuchi, Y. \& Funatsu, K. (2019). Consciousness of training body part(s) by showing a picture of various exercises in trunk muscles. International Journal of Sport and Health Science, 17, 135-147. https://doi.org/10.5432/ijshs.201915

Oshita, K. \& Yano, S. (2020). Influence of haptic sensory input through different kinds of clothing on gait performance. Applied Sciences, 10, 7590. https://doi.org/10.3390/app10217590

Oshita, K. (in press). Tactile cue by touching the agonist muscle increases the muscle activity during arm curl exercise. IFMBE Proceedings, 82 (accepted).

Schoenfeld, B.J. \& Contreras, B. (2016). Attentional focus for maximizing muscle development: The mind-muscle connection. Strength and Conditioning Journal, 38(1), 27-29. https://doi.org/10.1519/SSC.0000000000000190

Stramel, D.M., Carrera, R.M., Rahok, S.A., Stein, J. \& Agrawal, S.K. (2019). Effects of a person-following light-touch device during overground walking with visual perturbations in a virtual reality environment. IEEE Robotics and Automation Letters, 4(4), 4139-4146. https://doi.org/10.1109/LRA.2019.2931267

Vance, J., Wulf, G., Töllner, T., McNevin, N., \& Mercer, J. (2004). EMG activity as a function of the performer's focus of attention. Journal of motor behavior, 36(4), 450-459. https://doi.org/10.3200/JMBR.36.4.450-459

Zachry, T., Wulf, G., Mercer, J., \& Bezodis, N. (2005). Increased movement accuracy and reduced EMG activity as the result of adopting an external focus of attention. Brain research bulletin, 67(4), 304309. https://doi.org/10.1016/j.brainresbull.2005.06.035

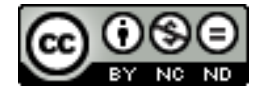

This work is licensed under a Attribution-NonCommercial-NoDerivatives 4.0 International (CC BY-NC-ND 4.0). 